\title{
Opportunities, Challenges and Use Cases of Digitization within the Semiconductor Industry
}

\author{
Germar Schneider \\ Infineon Dresden Technologies $\mathrm{GmbH}$ \\ Dresden, Germany \\ germar.schneider@infineon.com \\ Sophia Keil \\ Faculty of Business Administration and \\ Engineering \\ University of Applied Sciences Zittau/ \\ Görlitz \\ Zittau, Germany \\ sophia.keil@hszg.com
}

\author{
Gerhard Luhn \\ Innovation Management \\ Systema $\mathrm{GmbH}$ \\ Dresden, Germany \\ gerhard.luhn@systemagmbh.de
}

\begin{abstract}
Over the last years, Infineon Dresden Technologies has continuously been working on offering innovative products and enhancing the productivity of their wafer fabrication areas by increasing fab capacities as well as increasing automation in their $\mathbf{2 0 0}$ and $300 \mathrm{~mm}$ wafer manufacturing plants to improve productivity. One of the most important success factors is use of powerful automated material handling systems (AMHS) combined with the digitization of core processes within the whole company. Digitization supports converting a highly automated fab into a "smart fab" by horizontal and vertical integration of the internal supply chains. Within this paper, the authors provide an overview of three use cases for continuous improvement of the manufacturing process through use of automation and digitization to enhance the profitability of the semiconductor manufacturing plant.
\end{abstract}

Keywords-OHT, hybrid AMHS, conveyor, AGV, smart cam, big data

\section{INTRODUCTION}

Classic productivity levers like the use of an Automated Material Handling System (AMHS), lean management, or advanced dispatching and scheduling have been widely used in semiconductor fabrication facilities [1], [2], [3]. To achieve the next stage of operational effectiveness and efficiency, the end-toend digitization of all physical assets and its interaction with humans pursued within the Industry 4.0 initiative in Europe, offers huge opportunities and challenges for semiconductor manufacturers.

In this paper, we analyze and describe the opportunities and challenges by presenting the following three use cases for a high mix, high volume semiconductor fabrication facility:

- Use Case 1: Implementation of hybrid, self-controlled AMHS,

- Use Case 2: Implementation of intelligent algorithms to optically control the physical material flow in order to enhance product quality and

- Use Case 3: Automation of decision making process flows and holistic approaches using real time data and enabling high data quality.

The implementation of the three different use cases helps to improve all key parameters in a semiconductor fab. Use case 1 will improve the cycle time for R\&D lots as well as reduce the cycle time of production lots. Using intelligent algorithms within the material flow helps to assure the product quality at any stage within the manufacturing process. Lastly, big data handling and intelligent data management uncover completely new approaches that engineers and fab managers can apply to new challenges driven by increasing customer demands use to reduce administrative resources in an IC company.

\section{USE CASE I: IMPLEMENTATION OF HYBRID, SELF-CONTROLLED AMHS}

One major success factor for ensuring the competitiveness of mature semiconductor factories is the lot transportation system which essentially determines the cycle times of the products in a fab. During the set-up of new $300 \mathrm{~mm}$ factories, Overhead 
Hoist Transportation (OHT) systems are used almost exclusively. This provides high transportation speeds in the respective production bays, and since OHT systems are installed above the production equipment, this provides a relatively flexible layout. Over the last few years, a large number of innovative transportation solutions have been introduced to the market, and individual components provide technical innovations, higher flexibility and, in some cases, very favorable investment costs compared to expensive OHT solutions.

When establishing new factories such as the $300 \mathrm{~mm}$ power semiconductor line at Infineon Technologies Dresden (IFD), a large number of today`s existing AMHS components were compared and evaluated. Table 1 gives an overview of the most commonly used AMHS components in semiconductor fabs which are currently available on the market.

Table 1: Comparison of Different AMHS Components

\begin{tabular}{|c|c|c|c|c|}
\hline & Speed, CT & Shipping Volume & Flexibility & Costs \\
\hline OHT & ++ & + & - & -- \\
\hline SbT & ++ & + & - & - \\
\hline CTF & + & + & + & 0 \\
\hline RGV & 0 & 0 & + & + \\
\hline AGV & 0 & 0 & ++ & + \\
\hline PGV & -- & -- & ++ & ++ \\
\hline UTB & ++ & + & 0 & - \\
\hline Stocker & - & - & - & 0 \\
\hline
\end{tabular}

+t: very well, +: well, 0: neutral, - poor, -- very poor applicable

Five different methods that provide fully automated lot transportation (OHT, conveyor systems (CV), rail guided vehicles (RGV) and autonomous guided vehicles (AGV)) have been compared in terms of speed, volume, flexibility and cost of ownership. Furthermore, components like stocker systems used in many fabs and under track buffers (UTB) have been investigated. The overview shows that classic OHT systems are excellent systems for a fast loading and unloading of the semiconductor equipment in the different bays. However, those OHT systems require a high clean room ceiling and the components are very cost intensive. Another problem is that compared to conveyor based handling systems the capacity of the OHT system is poor. Therefore, one option is to enhance the capacity of an OHT system with the implementation of high volume conveyor based systems. IFD has already gained many years of experience with conveyor systems due to its use within IFD's $200 \mathrm{~mm}$ line for logic products [4]. The idea was now to integrate new transportation elements into an existing OHT system in the $300 \mathrm{~mm}$ clean room. Besides careful planning and performing different simulation studies for the material flow before and after the implementation of conveyor components, the main challenge was integrating the components into a currently operating factory control system. The problem is that standard components available from different automation companies use their own control systems and data structures. Therefore, many different additional operation concepts must be defined and installed by the automation experts in the semiconductor company. However, the upgrade of the system provides the benefit of a balanced material flow within the fab and also improves cycle time by performing material balancing during lot storage. Fig. 1 shows that in a standard semiconductor fab, the storage of the lots occupies roughly $70 \%$ of the entire manufacturing time (Material Flow Time in Fig. 1 is $82 \%$ of the 88 day cycle time).

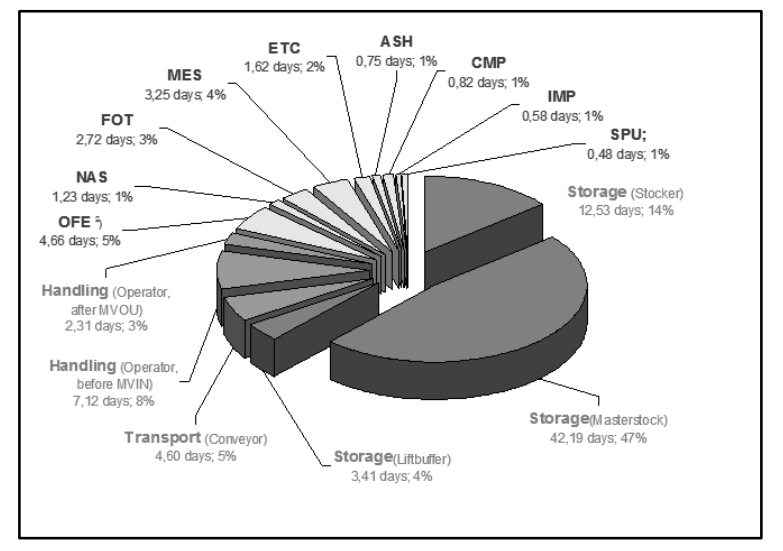

Fig.1. Lot processing and material flow times (storage, handling, transport).

The idea of so called "hybrid-AMHS" is to configure the whole system in a manner in which the storage time can be used to transport the lots in the direction of the next possible process steps and get the lots as close as possible to the next equipment. In this context, unified buffering systems (UTB) above the equipment are another very useful component for improving the whole system. A new upcoming trend in the high tech and logistics industry is the application of autonomous guided vehicles (AGVs), which is increasingly used at big logistics companies. 
The main advantage is that these systems can be flexibly used not only for one single transportation task but also for different transportation tasks. The goal was to create and add interfaces into a classic OHT based AMHS for AGVs. On the other hand, autonomous robotic systems must be controlled by the manufacturing execution system (MES) and the dispatching strategy is more complex compared to standard OHT systems. By using more than one robotic system, a fleet management system must be established for controlling the different robotic tasks. For this purpose, first concepts were developed on how a complete fleet of vehicles can be controlled to optimize lot dispatching in a semiconductor factory by using special algorithms. The algorithm which could be used is dependent on the complexity of the dispatching rules within different work centers. Therefore, a tool box was developed using simple or highly complex algorithms for optimized lot dispatching using AGVs. Depending on the product portfolio of the fab and the next ramp up steps to increase the fab capacity, a decision metric was worked out for the infrastructure of the next bays. The current use case at IFD focuses on a hybrid AMHS which can be extended flexibly and, in addition to the standard AMHS, enables further systems to be integrated into the Manufacturing Execution System (MES). Based on sufficient IT integration and a visualization of the system in real time, a hybrid system combines all the advantages of the respective transportation system components and is an important milestone for improving the factory performance. Fig. 2 shows a simple layout of how different components of an AMHS can be combined to create a hybrid AMHS.

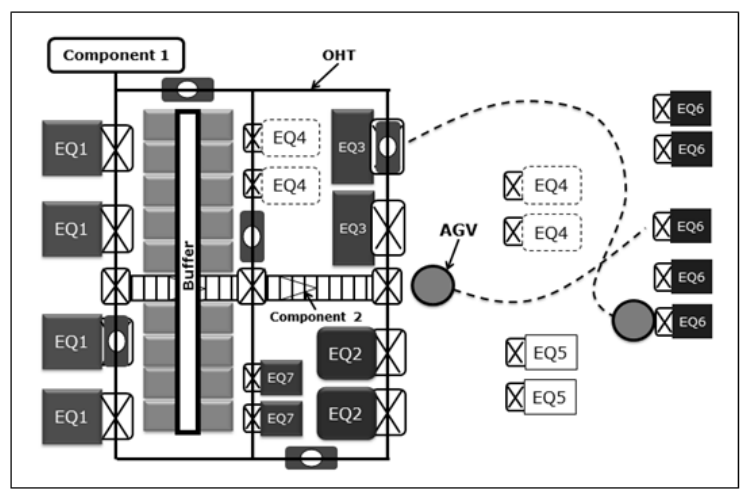

Fig. 2. Scheme of AMHS with new components (hybrid AMHS).
Currently, there is no literature available dealing with hybrid AMHS from the prospective of connecting different transportation and handling solutions. Therefore, the described use case and this article show for the first time how a very efficient AMHS with today's hardware components can be installed in an already existing $300 \mathrm{~mm}$ wafer fab.

\section{USE CASE 2: IMPLEMENTING INTELLIGENT ALGORITHMS CONTROLLING THE PHYSICAL MATERIAL FLOW AND ENHANCING PRODUCT QUALITY}

The next consecutive step after automation of the loading and unloading processes of the manufacturing equipment by using robot technologies is optical control of the material flow since automation unfortunately removed the ability of the human eye to observe material flow. After the successful implementation of fully automated systems, wafer loss within the process chains increased because of late feedback from subsequent defect density or electrical test. Today, digitalization offers the opportunity to visually monitor the flow of material using optical sensors, such as camera-based applications. New to the market smart camera systems can be installed easily in standard semiconductor equipment and offer very low cost opportunities compared to manual wafer inspection by operators in a fab. Another big advantage is that automated process control using smart cameras is an approach well defined and standardized by the engineers and offers constant and secure quality control for each wafer or lot. As a first step, the requirements for the quality control have to be defined. Secondly, an algorithm must be written by the process engineers. Today, a large number of algorithms are already available which can be used or simply adapted for monitoring and controlling material flows. These are characterized by low operating costs and extremely high availability and accuracy. One application in this use case is controlling the correct position of the wafers in the process equipment. Fig. 3 shows an example of how wafer counting can be performed with a smart camera located above the wafers. In addition to simply counting the wafers, misprocessing such as cross slotted wafers in a cassette or even broken wafers can be detected and the process can be stopped before 
crashing the wafers and leading to expensive tool down times.

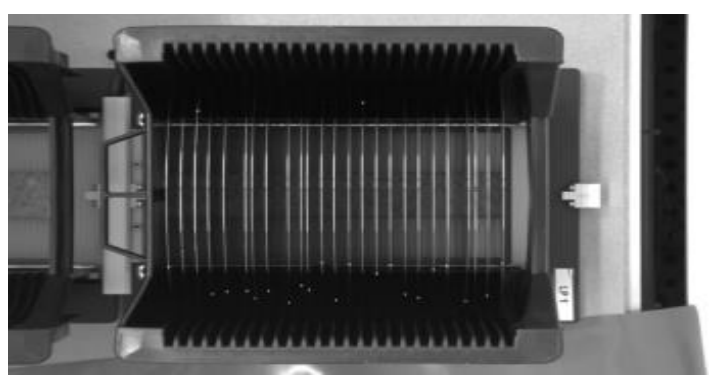

Fig. 3. Camera System used for wafer counting and quality control.

Moreover, the use of algorithms offers many more possibilities. Residues on the wafers or process deviations directly within the tools can also be controlled fully, thus automatically assuring stable outgoing yields in a fab. The most challenging task besides the programming of the specific algorithms is the integration into the MES. A standard based on OPC UA that defines how future smart cams can easily be connected in a fab will be developed. Fig. 4 gives an overview of the integration scheme of one application which was one of the first use cases within the IFD fab.

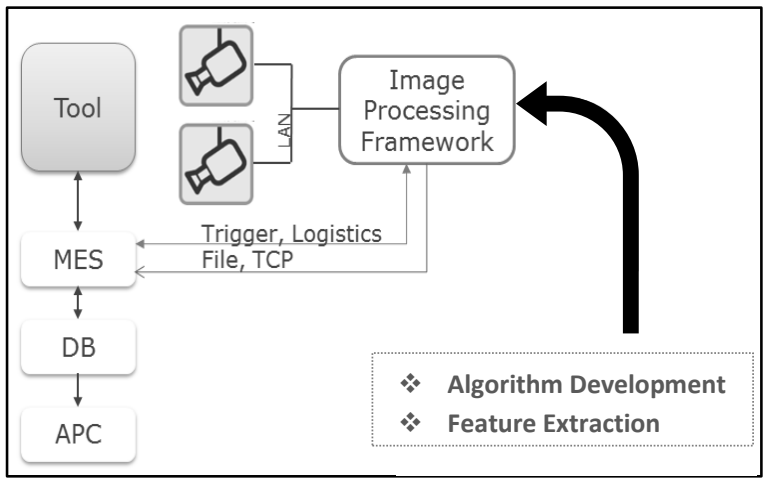

Fig. 4. Integration scheme of a smart cam.

Within the upcoming years many new already defined applications that use smart cameras for automated wafer will be demonstrated in the IFD fabs. Utilizing the full potential of those new applications is an important step on the way to improve the fab performance for existing and new factories supported by digitalization.
IV. Use CASE 3: Automation OF DeCision MAKING PROCESS FLOWS OF MANUFACTURING SUPPORT PROCESSES USING REAL TIME DATA AND ENABLING HIGH DATA QUALITY

As described in the introduction, today's $300 \mathrm{~mm}$ factories increased the degree of automation within the production area to more than $90 \%$, thereby both reducing costs and improving the quality of the products. Nevertheless, the automation led to a disproportionate increase in administrative resources which support production processes. This has the following effects:

- Additional requirements for highly qualified specialist personnel,

- High resource level required to manage the complexity of the different automation systems,

- Increased complexity of decision making, and

- Time delays during decision making.

Consequently, the next level of automation is to address administrative support processes for office employees (to reduce the required resources for repetitive administrative processes and the related costs, thereby enriching employee work time by providing time for innovative tasks). In the banking and service sector, the implementation of digitized workflows has already led to a considerable reduction in manual workload. These adjustments enable employees to use valuable time for customers and for value-add activities. Intelligent networking of various databases, including the possibility to have this data available in real time, can also be used to achieve this level in the semiconductor industry. Nevertheless, one of the biggest problems in the semiconductor business today is increasing complexity and an enormous increase in data which must be analyzed to support mass production. Therefore, ways must be found to optimize data handling, data storing and data calculation. Fig. 5 shows how intelligent data handling can be performed using a holistic data management system. 


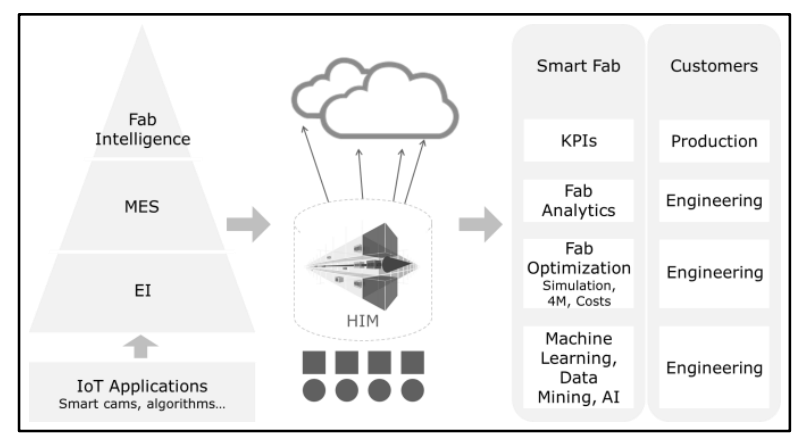

Fig. 5. The holistic data management concept.

New databases using important fab data (e.g. all existing logistic data of a fab which is used to control the fab as well as sensor data like described in Section III) can be connected using Internet of Things (IoT) applications to the Equipment Integration (EI) scheme of the fab and can later be used in artificial intelligence applications. Artificial intelligence creates the opportunity to automate business processes. The core of the new data management system is a holistic data structure where all the data is in a format offering fast calculation times and real time capability. Fig. 6 gives an overview of how the data is structured including the performance gain (typical quadratic complexity will be replaced by effective linear complexity).

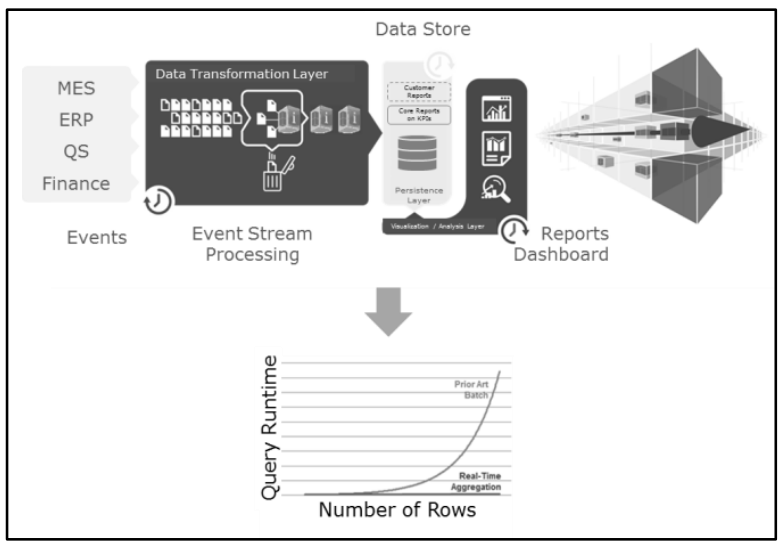

Fig. 6. Optimized Data Structures using the Holistic Approach (more details in [5]).

The first use case at IFD for the automation of administrative business process was the wafer start process. Five experts were previously needed to manually disposition production lots, R\&D lots, and non-production wafer starts each week. High manual effort was needed for manual checking and dispositioning different materials in different data bases. Some of the data was not even stored in a database and needed to be created by the experts each week. Therefore, the first objective was to define the data structure and the data bases to be used. The second step included identifying a weekly process to receive the data from only one algorithm. Fig. 7 shows the data and the data bases which are used week by week to define the weekly wafer start planning including media disruptions

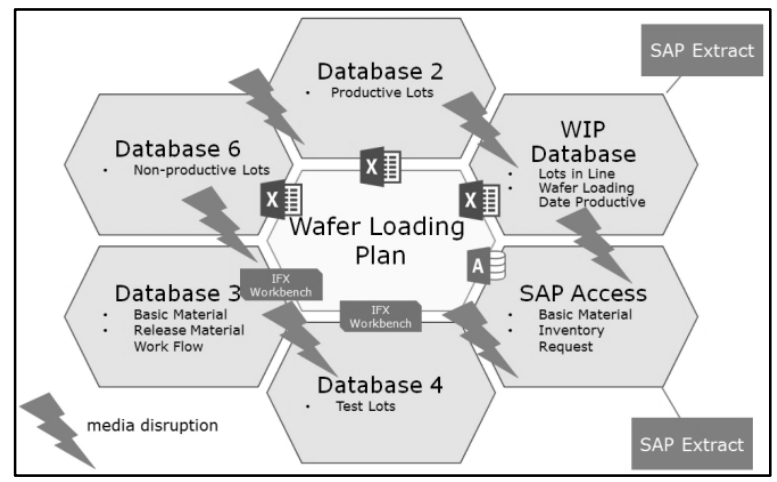

Fig. 7. Overview about databases used for the WSPW planning.

The overview showed that five separate databases are needed to manually establish the weekly wafer start plan and that the data bases are not linked to each other. Therefore, we had to find a way to overcome media disruptions. Finally, an algorithm able to collect the data and apply it to raw files needed to be found where only controlling and simple modification adjustments would be performed by the line control experts. The set-up of a new database structure and the implementation of the workflow automation helped to save more than $50 \%$ of time required to perform these business processes. This time could instead be used by the engineers to work on topics that create more value for the company. This is only one example of improvement in an office process. Transfer of this automation concept to other business processes in a semiconductor fab provides the potential to significantly reduce resource and cost. Besides the simple saving of headcount, the automation of those mundane steps will also be an enabler for continuous quality improvement by focusing resource on important core processes within the semiconductor manufacturing organization.

\section{CONCLUSION AND OUTLOOK}

The results of this work demonstrated by using three use cases show the potentials of novel applications in the field of digitalization within the semiconductor industry and their positive effects on key performance 
indicators of a wafer fab. Digitization paves the way for those applications, whereas digitalization can be utilized for exploiting the creation of value.

An already existing OHT-based wafer transport system was converted into a hybrid system and redesigned in a way that combines all the advantages of other transportation components like conveyor or UTB systems, thereby drastically improving throughput and cycle times.

In parallel to the automation of wafer transportation, quality assurance within a highly automated fab was secured with optical controlled procedures executed by "smart cams". It could be shown, that innovative algorithms can be implemented in production for specially tailored applications within wafer processing.

Another consequent step towards smart manufacturing is the use of big databases that are able to monitor the material flow or can even be used for automation of administrative processes. The holistic information method (HIM) holds the potential to bridge the growing gap between the ever growing amount of data and the non-trivial request to analyze and further exploit this data in real-time (thus to continuously strive for production optimization and valued learning in real-time).

The implementation of the results of the three use cases is an important step towards the conversion of a highly automated fab into a "smart fab". Based on the results, other projects will be started in order to develop a cross-fab "smart cluster" to control all processes in smart factory networks at any time in the world.

\section{ACKNOWLEDGEMENT}

A part of the work has been performed in the project Power Semiconductor and Electronics Manufacturing 4.0 - (Semi40), under grant agreement No 962466. The project is cofounded by grants from Austria, Germany, Italy, France, Portugal and - Electronic Component Systems for European Leadership Joint Undertaking (ECSEL JU).

\section{REFERENCES}

[1] H. Heinrich, S. Keil, G. Schneider, F. Heinlein, R. Lasch, and A. Deutschländer, "Pursuing the Increase of Factory Automation in $200 \mathrm{~mm}$ Frontend Manufacturing to Manage the Changes Imposed by the Transition from High-Volume Low-Mix to High-Mix Low-Volume Production", IEEE/SEMI ASMC, pp. 148-155, 2008.

[2] G. Schneider, T. Wagner, and M. Kraft, "Use of simulation studies to overcome key challenges in the fab automation of a $300 \mathrm{~mm}$ power semiconductor pilot line comprising thinwafer processing", IEEE/SEMI ASMC, pp. 42-47, 2015.

[3] S. Keil, D. Eberts, R. Lasch, and G. Schneider, "Managing Variability within Wafertest Production by Combining Lean and Six Sigma", IEEE/SEMI ASMC, pp. 32-38, 2012.

[4] H. Heinrich and A. Pyke, "The Impact of Conveyor Transports on Factory Performance at Infineon's (Siemen's) 200-mm Fab", Fabtech, Dec 1999.

[5] G. Luhn,, D. Habich, K. Bartl, J. Postel, J, T. Stevens, and M. Zinner, "Real-Time Information Base as key enabler for Manufacturing Intelligence and "Industrie 4.0"', IEEE/SEMI ASMC, pp. 216-222, 2015. 